\title{
Timing and tracking for the Crystal Barrel detector
}

\author{
Reinhard Beck ${ }^{1, \star}$, Kai Brinkmann ${ }^{2, \star \star}$, and Rainer Novotny ${ }^{2, \star \star \star}$ \\ ${ }^{1}$ Helmholtz-Institut für Strahlen- und Kernphysik, Rheinische Friedrich-Wilhelms-Universität Bonn, Nußallee 14-16, 53115 Bonn \\ ${ }^{2}$ II. Physikalisches Institut, Justus-Liebig Universität Gießen, Heinrich-Buff-Ring 16, D-35293, Gießen
}

\begin{abstract}
The aim of the project D.3 is the upgrade of several detector components used in the CBELSA/TAPS experiment at ELSA. The readout of the Crystal Barrel Calorimeter will be extended by a timing branch in order to gain trigger capability for the detector, which will allow to measure completely neutral final states in photoproduction reactions (see projects A.1 and C.5). Additionally, the readout of the inner crystals of the TAPS detector, which covers the forward opening of the Crystal Barrel Calorimeter, will be modified to be capable of high event rates due to the intensity upgrade of ELSA. Furthermore, a full-scale prototype Time Projection Chamber (TPC) has been built to be used as a new central tracker for the CBELSA/TAPS experiment at ELSA and the FOPI experiment at GSI.
\end{abstract}

\section{Introduction}

During the last beam times, the CBELSA/TAPS experiment has proven to be a reliable and stable detector system to measure polarization observables in different reactions with high precision (see project A.1). The previous measurements were mostly focused on the photoproduction of single or multiple neutral mesons off the proton in reactions like $\gamma p \rightarrow p \pi^{0}, p \eta$ and $p \pi^{0} \pi^{0}$. The measured data sets have been included in the partial wave analysis and are used to further constrain the calculations (see project A.2). However, most data sets used so far contain the proton in the initial state. Completely neutral reactions like $\gamma n \rightarrow n \pi^{0}$ pose a higher challenge for the detector system and only sparse data is available right now. Since the CBELSA/TAPS experiment is able to measure these reactions only with a very low efficiency, one of the goals of the project D.3 was the upgrade of the detector system to provide higher trigger capabilities (see section 2). Therefore the readout of the Crystal Barrel Calorimeter was extended by a timing branch.

Additionally, a higher photon rate in the experiment would be desirable, to enlarge the data set taken during a beam time. For the higher rates, an upgrade of the inner crystals of the MiniTAPS detector is necessary. These crystals are close to the photon beam line and are therefore hit by a high rate of particles. The upgrade of the crystals is described in section 4. To achieve a higher data rate in the crystals of the Crystal Barrel detector a new digitization stage of the energy branch is needed. Therefore a new Sampling-ADC (SADC) readout was developed, which is

\footnotetext{
^e-mail: beck@hiskp.uni-bonn.de

$\star \star$ e-mail: Kai-Thomas.Brinkmann@exp2.physik.uni-giessen.de

$\star \star \star$ e-mail: r.novotny@exp2.physik.uni-giessen.de
}

further shown in section 3 .

Another goal of the project D.3 is the development of a new central tracker, which could be inserted into the Crystal Barrel detector. A Time Projection Chamber (TPC) based on Gas Electron Multipliers (GEM) for charge amplification was built and the first prototype could be used as a tracker for the FOPI experiment at GSI. The detailed description of the new TPC and the gain calibration can be found in section 5 .

\section{Trigger Upgrade of the Crystal Barrel Calorimeter}

\subsection{Situation before the SFB}

The data acquisition of the CBELSA/TAPS experiment is built up in gated mode. This means that the experiment has a global trigger signal, which needs to be distributed to each module involved in digitizing data. The latency of this signal, i.e. the delay from the physical event to the leading edge of the trigger signal needs to fulfill the requirements of the existing read-out modules of the whole experiment and thereby is limited. The MiniTAPS modules allow for a maximum delay of $750 \mathrm{~ns}$. To achieve the allowed latency for trigger signals, the propagation delay on cables and the processing time of the central trigger module has to be taken into account. This results in a maximum latency of $500 \mathrm{~ns}$. Any signal which is supposed to be used in the trigger condition has to arrive at the central trigger module within this time.

In the former setup, the following detectors could be included in the trigger condition:

- The MiniTAPS Calorimeter, consisting of $\mathrm{BaF}_{2}$ crystals, which have a fast light output. 
- The Forward Conus, which is a small part of the Crystal Barrel Calorimeter in the forward region, upgraded with a fast readout.

- The Inner Detector, which consists of scintillating plastic fibers.

The fiber detector offers a high angular coverage, but is only sensitive to charged particles. MiniTAPS and the Forward Conus can additionally detect photons with a high efficiency and even neutrons with a limited efficiency. However, those only cover an angular range of $1^{\circ}$ to $27.5^{\circ}$ relative to the beam axis.

The remaining part of the Crystal Barrel Calorimeter was only included in the second level of the trigger. Therefore, an insufficient number of hits in the Crystal Barrel Calorimeter can abort the digitization of an event to save dead time. However, due to its long latency of $10 \mu$ s, the hit information cannot be used to start the acquisition process.

This results in a limited trigger efficiency for reactions with purely neutral final states, like $\gamma n \rightarrow n \pi^{0}$. The result of a simulation of the current efficiency is shown in Fig. 1.

\subsection{Upgrade within the SFB}

The goal of this subproject was to modify the existing readout of the Crystal Barrel Calorimeter, or to build a new one, which generates hit signals fast enough to be included in the first level of the experiment trigger. The most obvious approach - extending the readout of the Forward Conus to the remaining calorimeter - is not applicable: the Forward Conus uses photomultipliers to convert the scintillation light into an electrical signal. As these are not operational in strong magnetic fields, such a readout would prevent the magnet upgrade which is planned in extension of the TPC upgrade.

\subsection{Challenges of extracting fast timing information from CsI:TI}

As seen in section 2.1, the hit information of the calorimeter has to be present at the central trigger module within $500 \mathrm{~ns}$ after the physical event in the target. This is conflicting with the scintillation properties of the CsI:Tl crystals of the Crystal Barrel Calorimeter. The light emission has a decay time constant of $\tau=1 \mu \mathrm{s}$. This means that only a fraction of the light has been emitted, when the hit information has to be present latest. Furthermore, the full 500 ns cannot be used to accumulate the signal. Within that time span, also signal processing and transmission has to take place. The signal processing includes the evaluation of the number of clusters in the whole calorimeter from the hit pattern of each single crystal (cluster finding). Assuming a time span of $150 \mathrm{~ns}$ for the signal accumulation results in only $\sim 10 \%$ of the full signal.

Moreover, the crystals are large compared to a typical photodetector. This means that the scintillation light in average has to be reflected many times inside the crystal before it can be absorbed in the photodetector. This allows photons to be absorbed in passive material, before it can reach the photodetector and contribute to the output signal. The light collection of the finally used readout was measured to be 3000 Photons/MeV [10]. This is an increase compared to the previous readout of 2000 Photons/MeV [11], but small compared to the total amount of light (54000 Photons/MeV [12]).

So, although CsI:Tl is known as a very bright scintillator, the readout has to be able to process comparatively small scintillation signals. Furthermore, it has to be able to process the fully accumulated scintillation signal, as this is used for the energy determination.

The time span actually used for the accumulation of the scintillation signal results from the design of the readout and has to be chosen carefully. It should be large enough to yield a sufficiently high signal for a given energy deposit. On the other hand, the hit information has to fit into the existing trigger scheme. A too short time span will result in poor signal strength, which will increase the detection threshold for the hit information. A too large time span might result in a loss of hit information of other detectors in the experiment.

\subsection{Implementation}

Three approaches have been evaluated during the SFB:

1. Keep the existing readout and extend it by a timing branch, which uses signal filters that generate fast timing signals.

2. Use the above mentioned filters, but also replace the existing readout with one based on avalanche photo diodes.

3. Extend the front-end with silicon photomultipliers, to get fast signals.

Options 1 and 3 resulted in either too high dark count rates or too high detection thresholds and were therefore discarded. Option 2 was developed into a final solution and elaborately tested on prototypes at accelerator facilities. The solution and the tests will be described in the following sections.

\subsubsection{The new Front-end}

The new front-end consists of two avalanche photodiodes (APDs), a charge sensitive preamplifier, a high voltage supply circuit, and a line driver.

\section{Avalanche Photodiode}

The front-end utilizes two APDs of the type S11048(X3) from Hamamatsu, operated at a gain of 50. Each APD has an active area of $6.8 \times 14 \mathrm{~mm}^{2}$ and has been characterized for temperature and voltage dependence [14]. The average coefficients were found to be $\alpha_{T}=-2.28 \% / \mathrm{K}$ and $\alpha_{V}=2.94 \% / \mathrm{V}$. The temperature coefficient varies in the range $\alpha_{T}=-2.16 \ldots-2.50 \% / \mathrm{K}$ and the voltage coefficient in the range $\alpha_{V}=2.80 \ldots 3.35 \% / \mathrm{V}$. The main part of the distributions have standard deviations of 


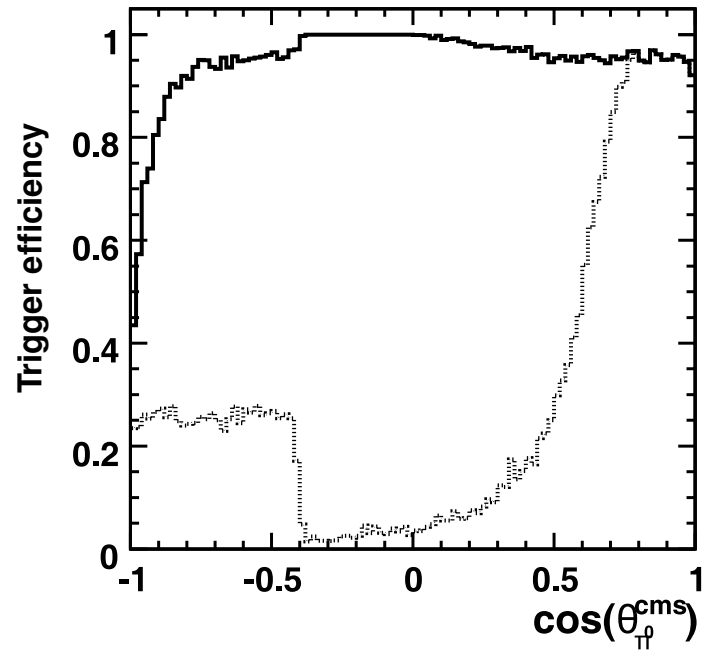

(a) Photonenergy $E_{\gamma}=700 \mathrm{MeV}$.

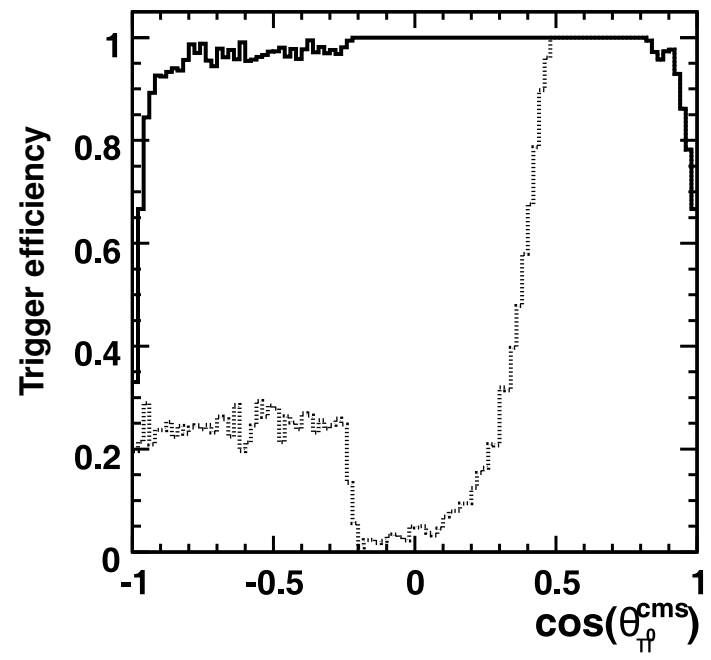

(b) Photonenergy $E_{\gamma}=2 \mathrm{GeV}$.

Figure 1. Trigger efficiency for the reaction $\gamma N \rightarrow N \pi^{0}$, for different photon energies. Dashed line: Efficiency for neutrons. Solid line: Efficiency for protons. [9]

$0.08 \% / \mathrm{K}$ and $0.08 \% / \mathrm{V}$ respectively [14]. The dark current was specified by the manufacturer to be in average $I_{D}=14.8 \mathrm{nA}$, with a total span of $5.6 \mathrm{nA} \leq I_{D} \leq 33.7 \mathrm{nA}$ at $25{ }^{\circ} \mathrm{C}$. The front-end utilizes two instead of one APD to improve the energy resolution below $10 \mathrm{MeV}$ energy deposit, to lower the threshold in the timing channel, and to enhance failure safety by redundancy.

\section{Preamplifier}

Each of the two APDs has its own preamplifier, which is a charge sensitive design. The amplification is $G=$ $147 \mathrm{~V} / \mathrm{nC}$, which results in a dynamic range of up to $E_{\max }=3.7 \mathrm{GeV}$ for the crystals with the highest light yield.

The decay time constant was chosen to be $\tau=54 \mu \mathrm{s}$, which is short enough to not cause extensive pile-up on the output signal and long enough to not degrade the signal strength due to ballistic deficit.

The preamplifier was originally developed for the PANDA experiment at the University of Basel and adapted for the usage in the Crystal Barrel Calorimeter. The printed circuit board (PCB) is manufactured in flexrigid technology. The amplifier circuit and the APDs are soldered on rigid parts, which are connected by a flexible part, which is a necessary feature during the assembly process. One of the 1300 produced new preamplifiers is shown in Fig. 2.

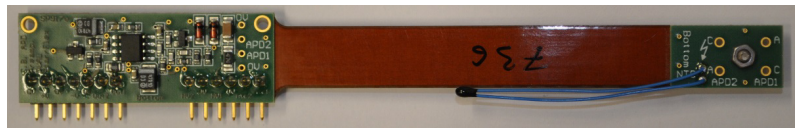

Figure 2. The new APD preamplifier.

\section{High Voltage Supply Circuit}

To enable the internal gain mechanism, the APD needs an external bias voltage in the order of $V_{B}=400 \mathrm{~V}$. Due to variations in the manufacturing process, each APD has a different bias voltage at which the desired gain of 50 is reached. One way to not need a unique high voltage channel for each APD is to group them. However, it was unknown how the desired bias voltage changes due to aging and radiation damage, which could result in the desired bias voltage of a group drifting apart by time. Therefore, another approach was used.

Each front-end module is supplied with a fixed high voltage of $500 \mathrm{~V}$. This voltage is reduced to the desired value in the front-end, independently for each APD. The voltage is set using a digital potentiometer, which can be remotely controlled ([15]) in the experiment setup and can be tuned in the range of $328 \mathrm{~V} \leq V_{B} \leq 458 \mathrm{~V}$ in steps of $\Delta V=127 \mathrm{mV}$.

To address the problem of the large temperature coefficient of the APD's gain, one implemented measure amongst others is a gain stabilization circuit, which is part of the HV supply circuit. This circuit utilizes an NTC to measure the temperature and to change the bias voltage in such a way that the gain change due to temperature change is counterbalanced.

A 16 Bit ADC allows to monitor the output voltages and the temperature. The HV supply card is shown in Fig. 3.

\section{Line Driver}

The signal is differentially transmitted to the back-end. The line driver circuit converts the preamplifier signal to a differential signal. Additionally, the gain of the driver circuit is adjustable in 16 steps to address the variation of the light yield of the crystals. The span between the brightest 


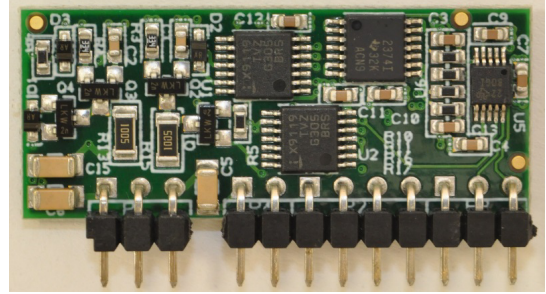

Figure 3. The new High Voltage Supply Card.

and darkest crystal was known to be around a factor of 2.5. By setting the gain of the line driver to the proper value, the dynamic range on the transmission lines is equalized to $E_{\max }=2.5 \mathrm{GeV}$

For each two preamplifiers in the font-end of one crystal, only one signal is transmitted. Therefore, the preamplifier signals need to be multiplexed. The multiplexer circuit outputs the average of both APDs by default. The averaging reduces the electronic noise of the front-end. Additionally, the multiplexer can be configured to output only the signal of one of the APDs, which increases the reliability, in the case that one of the preamplifiers is broken and outputs a bad signal.

Also, this feature turned out to be very valuable to check the quality of the optical contact between APD and crystal in the assembly process.

\subsubsection{The new Back-end}

The back-end consists of a timing branch, which provides the fast trigger information as well as timing data, and an energy branch, which is used to measure the energy deposit in the crystals. In the first step of the upgrade, the existing energy readout will be retained. In the second step, the existing QDCs will be replaced by SADCs, which are currently in development. The timing branch has to be fully built up.

\section{The Existing Energy Branch}

The current energy branch uses signal filters which output pulses with a peaking time of $\sim 3 \mu$ s for the CsI:Tl preamplifier signals. The pulse area is digitized with LeCroy $1885 \mathrm{~F}$ dual range QDCs.

\section{The New Timing Branch}

The main parts of the timing branch are a signal filter and a discriminator. The signal filter is used to achieve signals with a rise time, which is short enough to allow for integration into the first level trigger. The discriminator converts the analog timing signal into a digital hit information. The custom designed modules also include TDCs and a part of the cluster finder electronics.

\section{Timing Filter Modules}

The timing filters are $\mathrm{CR}(\mathrm{RC})^{2}$ filters. The low pass is implemented in a dual feedback topology. In most prototypes, the time constant was chosen such that the peaking time of the step response is $80 \mathrm{~ns}$. In the final design, this time was increased to $100 \mathrm{~ns}$ to further increase the signal to noise ratio.

The so achieved timing signal is differentially transmitted to the discriminator module. An additional output allows for a further extension of the module by an analog summation circuit. This is planned to be used as a fast energy sum of the whole calorimeter.

To connect the energy readout, the module has two buffered outputs which forward the preamplifier signal. As there are two outputs available, the upcoming SADC can be operated in parallel to the existing QDC in a commissioning phase.

One PCB covers 23 channels which reflects the detector geometry of 30 subsections of 23 channels in each half (Fig. 4). Two PCBs are housed in a double width NIM cassette.

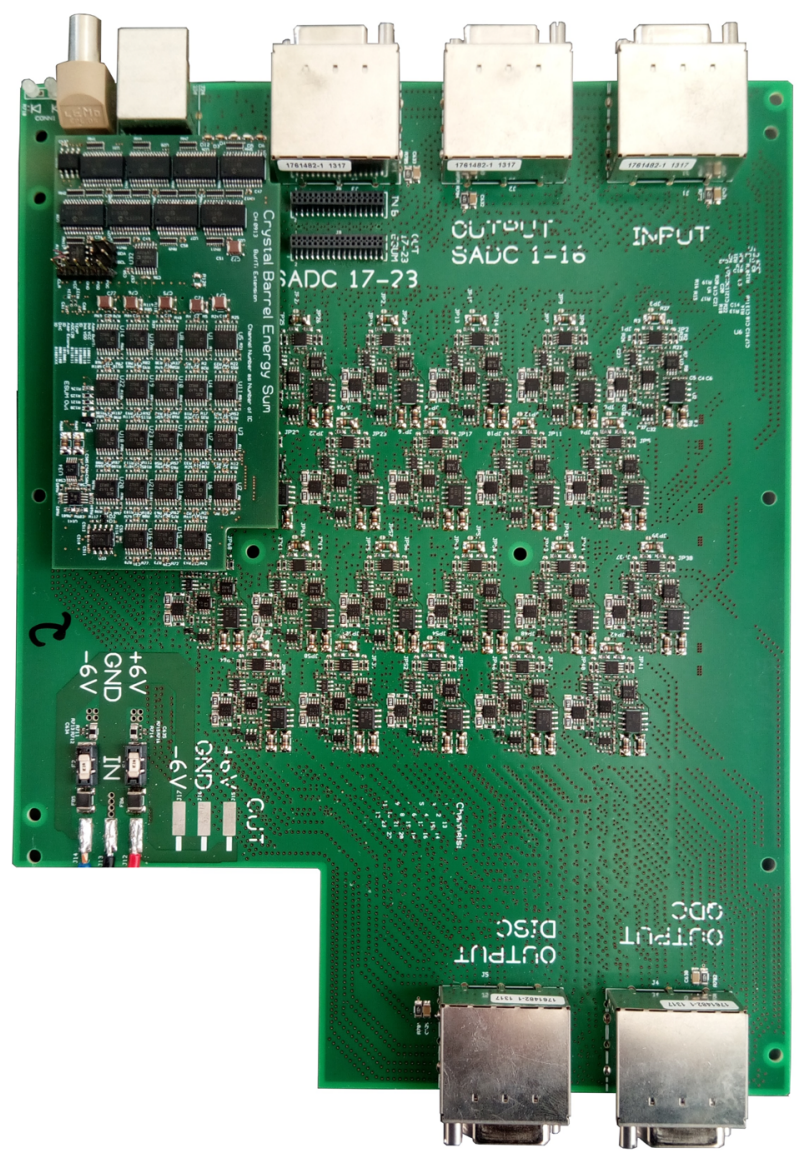

Figure 4. Timing filter module with distribution to the QDC/SADC boards.

\section{Discriminator}

The discriminator is a VME-Module. It contains 92 channels corresponding to 4 subsections of the calorimeter. Each channel has 2 comparators which allow for a time walk correction in the module. A Spartan 6 LX150 FPGA processes the comparator signals. A time information is extracted and as well as the first part of the clusterfinding is processed here (Fig. 5). 
MRJ21 connectors are used to achieve the required high input connector density. The 184 TDC channels are implemented utilizing the SERDES2 primitives on the Spartan 6 FPGA. Thereby a sampling rate of 800 MSPS is achieved, corresponding to a binning of $1.25 \mathrm{~ns}$ [16]. The resolution achieved is appropriate for the expected time resolution of the fully upgraded detector (see Sec. 2.5.3).

To use this module also on detectors with a much better time resolution, an existing FPGA TDC based on the carry chain approach [17] was adapted to the discriminator design and evaluated. A Time resolution of $\sigma_{t} \approx 30 \mathrm{ps}_{\mathrm{RMS}}$ was measured for sufficient sharp signals [18] Pulses with a width of 1.2 ns (FWHM) were shown to be reliably detectable, although the threshold has to be set to lower values than the amplitude of the pulse [18].

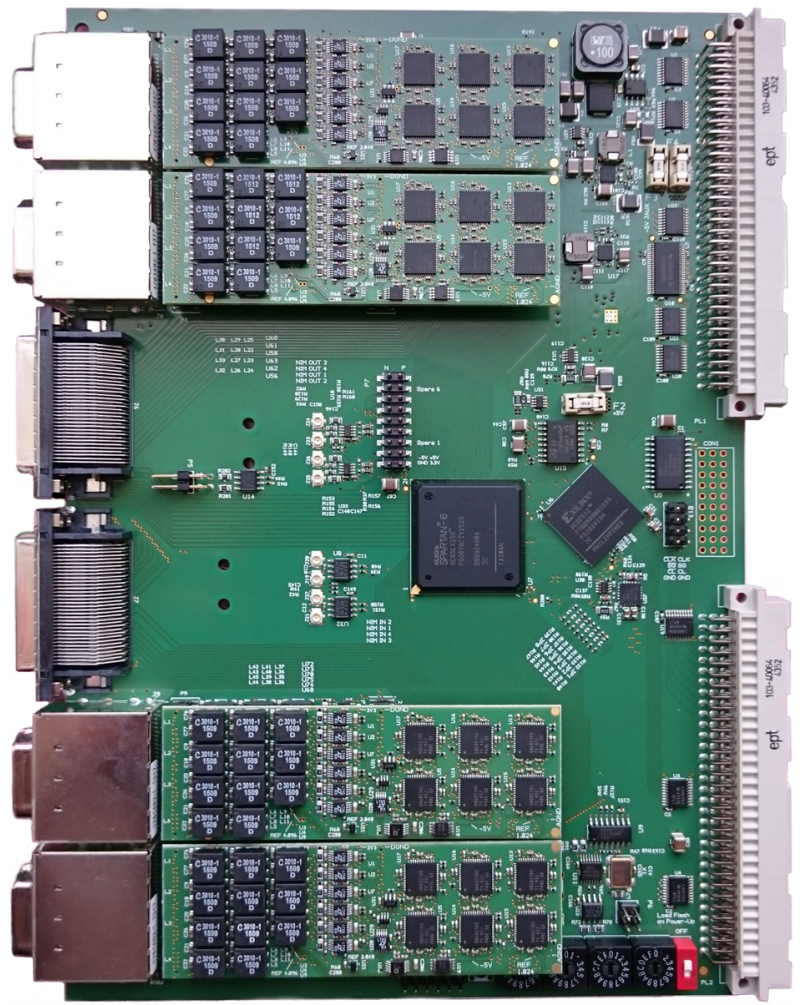

Figure 5. Discriminator board with first part of the cluster finder.

\subsubsection{Light Pulser System}

The former experiment setup used a light pulser system to perform a calibration of the high range versus the low range of the QCDs in the energy readout. This system had issues like low rate capability of a few $\mathrm{Hz}$, a rate dependence of the flash intensity, and a maximum intensity which did not allow to drive the QDC up to the highest channels.

The new light pulser system [14] utilizes a LED instead of a discharge bulb. The color of the LED matches the scintillation light wavelength. 6 LEDs with a power of $5 \mathrm{~W}$ each are used for the whole calorimeter. The custom developed driver circuit was tested with flash rates up to $f=20 \mathrm{kHz}$ [14], and a maximum energy equivalent of
$E_{\mathrm{Eq}, \max }=6 \mathrm{GeV}$. The flash intensity can be set using a DAC in the driver circuit. A preliminary analysis found the rate dependence of the intensity to be less than $2 \%$ [14], over the range from $2 \mathrm{kHz}$ to $5 \mathrm{kHz}$. A more precise determination is ongoing.

\section{APD Gain Monitoring}

Additionally to the QDC calibration, the light pulser system will be used for another purpose which is actually the main purpose of the upgrade: it will be used to measure the gain of the APDs during data taking in a photoproduction measurment.

The gain $G$ will be calculated using the quotient of the intensities with applied bias voltage $A_{\mathrm{HV}} \mathrm{ON}$ and with bias voltage set to zero $A_{\mathrm{HV}} \mathrm{OFF}$.

$$
G=\frac{A_{\mathrm{HV} \mathrm{ON}}}{A_{\mathrm{HV} \mathrm{OFF}}}
$$

A necessary key feature of the APD is that the temperature dependence of the signal strength is negligible compared to the temperature dependence of the light yield of the scintillation process in the calorimeter crystals.

As the temperature coefficient at zero bias condition is negligible, this operating mode is used as a reference point. Thus, changes in the light pulser system, like a changed transmission strength of the light fibers, can be cancelled out by repeating the reference measurement. It is planned to run the light pulser system during data taking in bias on mode and perform reference measurements only if necessary. To keep the light pulser as stable as possible, the system has been built up in a temperature stabilized environment. Thereby the brightness temperature coefficient of the LED of $0.25 \% / \mathrm{K}$ is addressed [14].

Another property of the APD makes the reference measurement more challenging: the parasitic capacitance of the APD is increased to roughly $C_{P}=7 \mathrm{nF}$ [14] at zero bias condition. In addition to the fact that the signal is smaller as the gain of the APD equals one, this introduces two more problems. First, the noise level is increased, as the capacitance of the APD is a directly influencing factor. Second, the signal strength is decreased even more: the charge sensitive preamplifier decouples the signal from the high potential of the APD's cathode using a capacitor. The signal charge is effectively divided between the input decoupling capacitance of the preamplifier and the parasitic detector capacitance $C_{P}$.

In the produced design, the decoupling capacitance is chosen to be $47 \mathrm{nF}$ to minimize the effect. The capacitance under operating conditions is reduced to $17 \mathrm{nF}$ due to the DC bias effect, which results in limiting the signal loss to $30 \%$. The high intensity capability of the new LED light pulser system allows to achieve sufficient signal to noise ratios despite the increased noise level.

\subsubsection{Ultra Fast Cluster Finder}

The former cluster finder was implemented on ASICs. The achieved latency is $\Delta t=0.8 \mu \mathrm{s}+n \cdot 0.8 \mu \mathrm{s}$, where $n$ is the number of clusters. In the former operation, the result of 
the encoder was evaluated $10 \mu$ s after the first level of the trigger had fired. To include the Crystal Barrel Calorimeter into the first level of the trigger, this process has to be improved to give a result within the available latency of $500 \mathrm{~ns}$, sharing that time span with the signal generation in the signal filters, the signal processing in the discriminators, as well as the signal distribution.

To fulfill this constraint, an entirely different encoding algorithm was chosen. Instead of latching the hit pattern and successively identifying the clusters in it, the new algorithm processes the whole hit pattern in parallel. Key idea of this approach is to use a certain hit pattern to identify one cluster, instead of determining connected entries in the hit pattern, like the former algorithm did.

The demanded hit pattern is shown in Fig 6(a). Each box represents a crystal. To be identified as a cluster, the crystal drawn in green has to have a hit, while the crystal drawn in red must not have one. Other crystals are not evaluated for this position. To cover the whole calorimeter, the pattern has to be checked on all possible positions on the detector matrix.

As the pattern only evaluates the local neighborhood of a crystal, it is not necessary to have the full hit information stored in one unique module. The contrary is the case: the algorithm allows by definition to split the encoding in subsectors and to distribute their processing over multiple modules. Only the hit information on the borders has to be exchanged between the processing units.

In the second stage, the cluster information, which is encoded in over 1000 yes/no decisions, has to be processed into a number which represents the number of present clusters. This is done by an adder in hierarchic structure. In each step, the number of interim results is halved, until after 11 steps one single number is present.

Another advantage is that no latching of the hit pattern is required as it was in the former algorithm. Implemented in a pipeline architecture, the algorithm can operate free running and provide a new number of clusters each clock cycle.

The algorithm is being implemented in the discriminator modules, as far as possible regarding the available hit data. The preprocessed information will be transmitted to a central module where the number of clusters is finally determined. According to the current development status, a total latency of less than $100 \mathrm{~ns}$ seems achievable.

Simulations have been performed to analyze the potential of the algorithm. The number of clusters found is shown in Fig. 7. The comparison to the former algorithm shows that in few cases a larger number of hits is found, while a lower number is in no case found. So, compared to the former algorithm, the sensitivity is at least the same, while the selectivity is slightly worse.

The supernumerary clusters are believed to originate from complex shaped clusters.

Other studies have shown that the new algorithm is also able to detect overlapping clusters to some degree. Therefore the number of detected clusters was compared to the number of particle energy deposits found in the full analysis. [16]

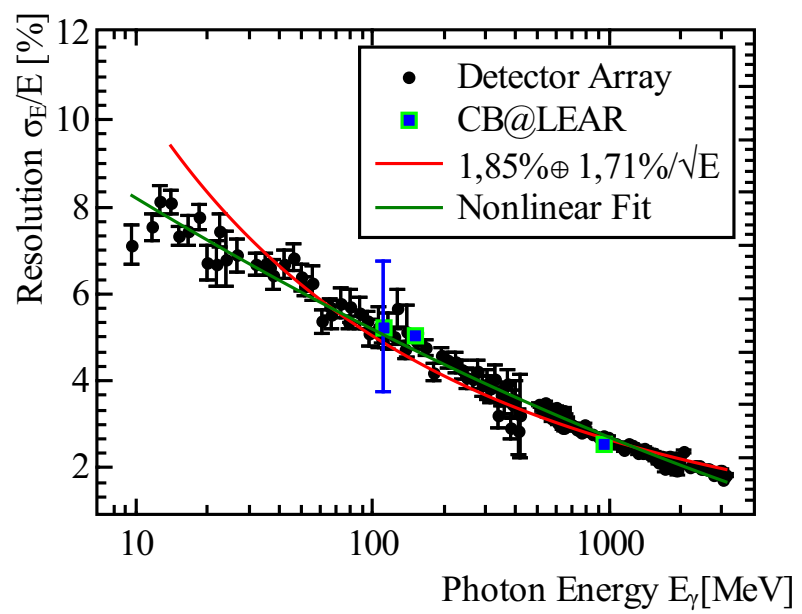

Figure 8. Measured energy resolution of the new and the former readout. The curves represents fits to the data [20].

\subsection{Prototype Evaluation}

In total, four generations of prototypes of CsI:Tl crystals with APD readout have been built up within the SFB. In this section, major results will be shown.

\subsubsection{Test Setup and Test Sites}

To be able to perform conclusive measurements on a photon beam, a $3 \times 3$ array of detector crystals from the Crystal Barrel Calorimeter has been built up. The array resembles a sub unit of the whole calorimeter. As only one size type of the crystals was available, the array is not perfectly congruent to any sub unit. Simulations showed that around $90 \%$ of the electromagnetic shower deposits its energy in the active material in the relevant range of incident photon energy.

To determine the energy and time resolution, measurements at the accelerator facilities MAMI and ELSA have been performed. The array was positioned in the photon beams of the A2 and the CBELSA/TAPS experiments. The tagging systems have been used to obtain the energy information of the incoming photons, as well as their time. Measurements have been performed at incident electron energies of $E_{e}=$ $180,450,800,1500,2400$, and $3200 \mathrm{MeV}$. This allowed to investigate the detector performance for photon energies in the range $9 \mathrm{MeV} \leq E_{\gamma} \leq 3.1 \mathrm{GeV}$.

\subsubsection{Energy Resolution}

The determined energy resolution is shown in Fig. 8 as black circles [20]. Blue squares represent the energy resolution that was found with the former readout, when the calorimeter was installed at LEAR.

The red curve represents a fit to the data:

$$
\frac{\sigma_{E}}{E}=\frac{(0,68 \pm 0,19 \%)}{\sqrt{E / \mathrm{GeV}}} \oplus \frac{(2,56 \pm 0,11 \%)}{\sqrt[4]{E / \mathrm{GeV}}} .
$$




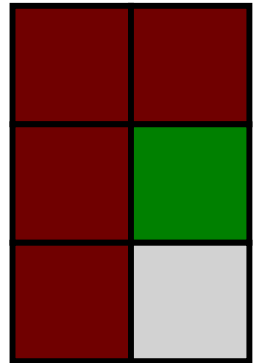

(a) Demanded hit pattern. Dark green: Hit demanded, dark red: hit must not be present, gray: not evaluated.

Figure 6. Illustration of the new cluster finder algorithm.

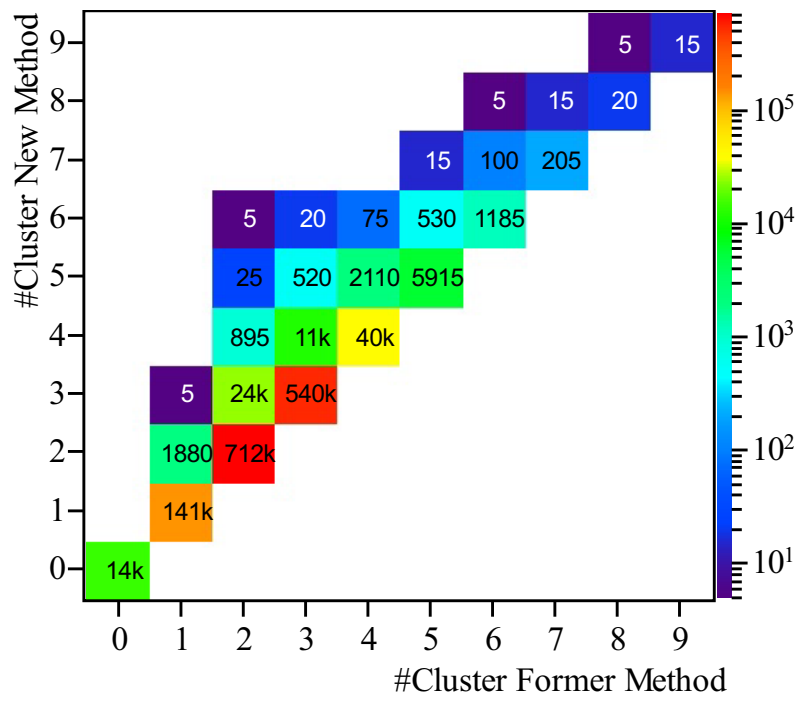

(a) $p \pi^{0}$

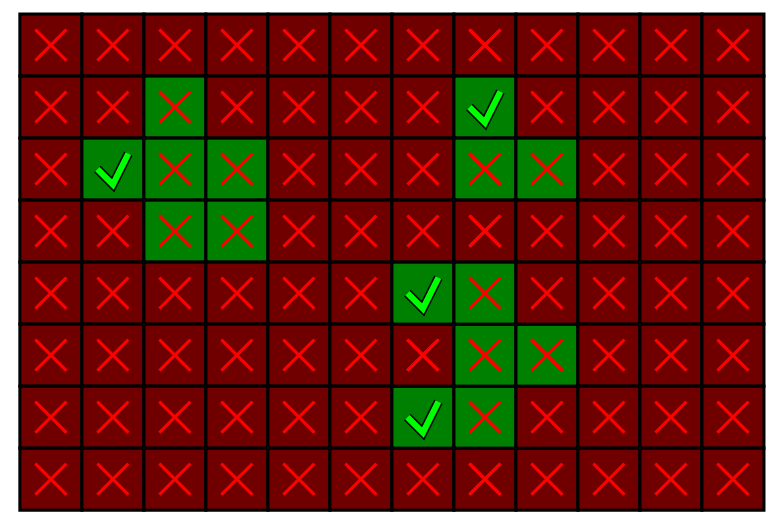

(b) Example hit pattern. Fulfilling of pattern shown in Fig. 6(a) denoted by green tick, not fulfilling by a red cross. The cluster on the lower right is mistakenly detected as two.

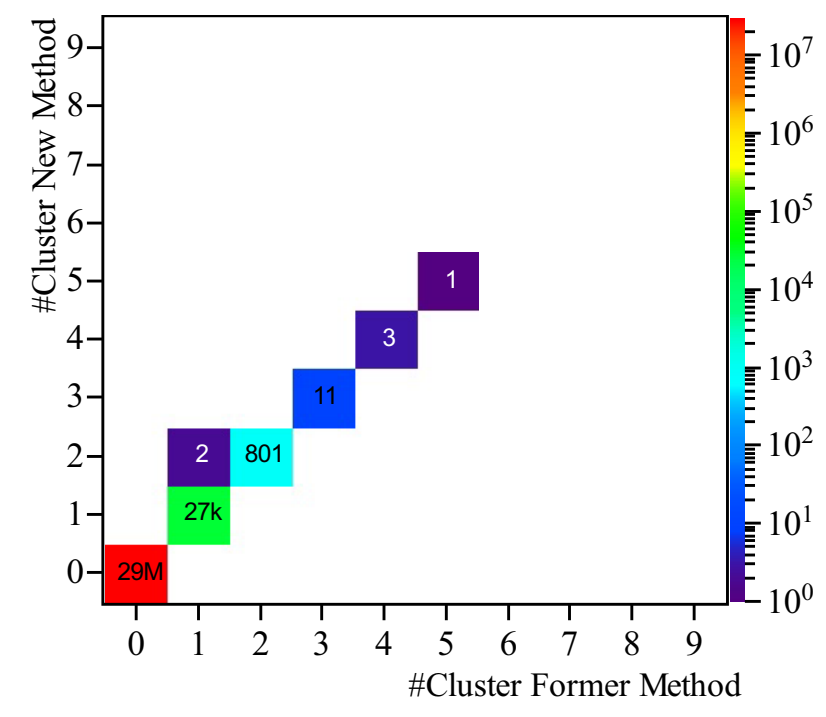

(b) Electromagnetic Background Reactions

Figure 7. Number of identified clusters using the former and the new algorithm. Data from Monte Carlo Analysis. [8]

The phenomenological $E^{-\frac{1}{4}}$ term was added to get a more precise description of the data. This term is also used for other CsI:Tl calorimeters to parameterize the energy resolution, and was the only term in an earlier publication about the Crystal Barrel Calorimeter [11]. A further constant term and one proportional to $1 / E$ were compatible to zero in the fit. If the energy resolution needs to be parameterized for practical reasons, like applying energy dependent cuts, it seems more reasonable to use the physically unmotivated parametrization $\frac{\sigma_{E}}{E}=0.117-\frac{0.01657}{\log (E / \mathrm{MeV})}+$ $\frac{0.0005}{\log ^{2}(E / \mathrm{MeV})}$, which is shown as a green line in Fig. 8 .

\subsubsection{Time Resolution}

The time resolution was extracted from the same data, which was used to determine the energy resolution. There are few differences one has to be aware of. While the energy of all crystals in one event is summed up for the de- termination of the energy resolution, crystals are treated independently to extract the time resolution.

The time resolution can be determined for energies much below the low energy end of the tagged photon range, as the electromagnetic shower distributes the energy of the incident photon among multiple crystals.

For each event, the energy entry of each crystal is saved as well as the time information if available. The hit signal of the tagger hodoscope was used as time reference.

The trigger condition during the data acquisition at the photon beams was:

\section{(hit in any crystal) AND (hit in the tagger).}

Fig. 9(a) shows an example of the time information of one crystal in dependence of the energy deposit in that crystal.

The vertical width of the distribution results from the accuracy of the time information, while the energy dependence 


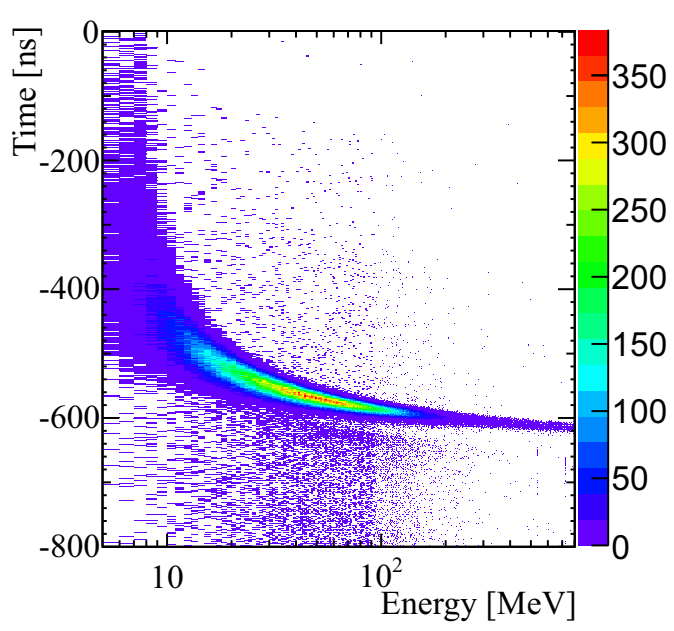

(a) Time information in dependence of energy deposit.

Figure 9. Diagrams on the time resolution [20].

of the mean time at a fixed energy results from the walk. As the vertical width of the distribution is energy dependent, the time resolution was determined in dependence of the energy. To achieve the standard deviation from the data, the Novosibirsk fit function was fitted to the data. This function implements a parameter $\tau$ characterizing the asymmetry of the distribution. For $\tau \rightarrow 0$, this function approaches the Gaussian distribution. The function was chosen regarding the slight asymmetry of the time information distribution. Fig. 9(b) shows the data of one energy bin. The used fit is shown in red. The fit of a Gaussian distribution is shown in green for comparison.

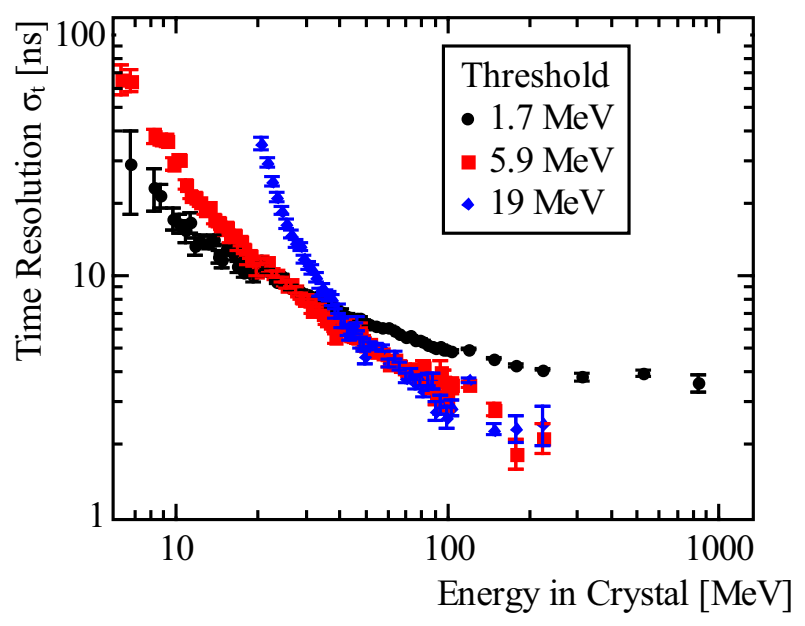

Figure 10. Time resolution at different thresholds in dependence of the energy deposit [20]

Fig. 10 shows the time resolution $\sigma_{t}$ of a typical crystal. The resolution improves with the energy deposit by more than an order of magnitude and reaches values around $\sigma_{t}=2 \mathrm{~ns}_{\mathrm{RMS}}$.

The threshold of the discriminator has not only influence on the minimal detectable energy, but also on the time

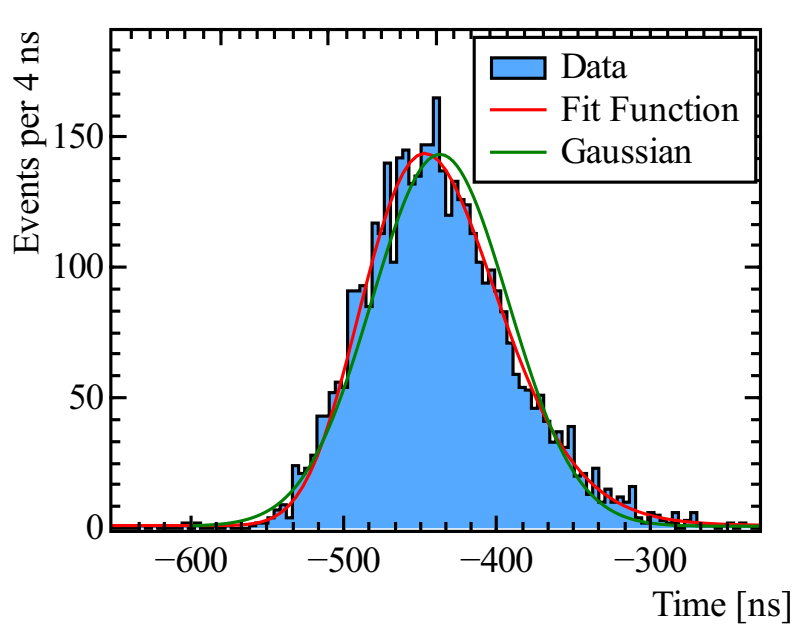

(b) Time information for a fixed energy with fits.

resolution. The resolution improves significantly at low energies if the threshold is lowered.

The custom build discriminators utilize two thresholds to compensate for walk of the timing signal. The simplest compensation approach is to forward the information of the lower threshold to the output, but only if the higher threshold was crossed within a given time limit after the lower one was crossed. By this, only pulses are forwarded which have more than a minimum steepness between the thresholds, which corresponds to rejecting pulses below a certain energy. This energy is defined by the thresholds and the allowed delay. In Fig. 9(a) it is visible, that the major time shift of the walk is present at low energies. Also the worst time resolution is found in the low energy

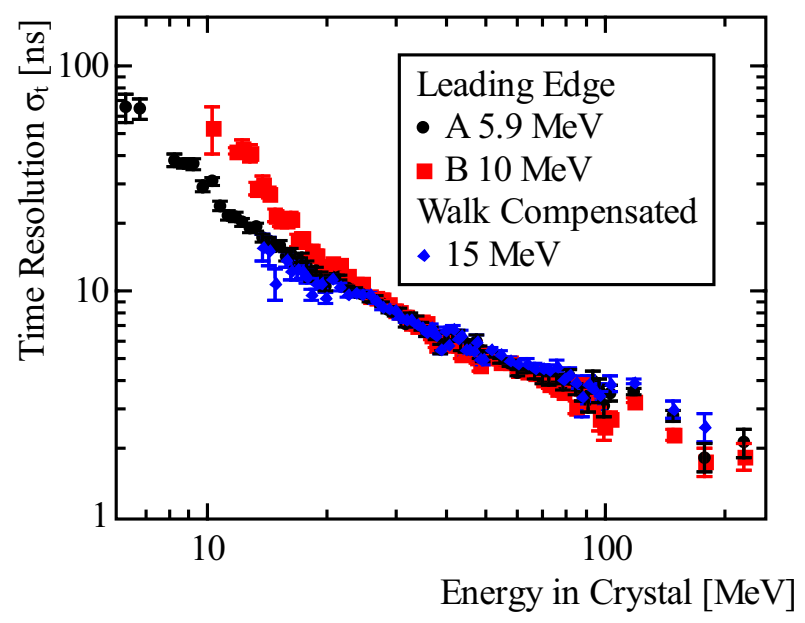

Figure 11. Time resolution of two thresholds and the resolution of the compensated signal (blue), which is generated from two thresholds (black, red) [20].

region. The timing signals will be used in the trigger using a coincidence. Therefore, the whole variation of the information has to be taken into consideration when the coincidence window is set. 
The compensation algorithm offers two advantages. First, low energy hits which have the worst timing resolution, are discarded (see Fig. 11). Second, the time resolution just above the detection threshold is better for the compensated information than for a leading edge discriminator having the same threshold. This can be seen in Fig 12 in the range $15 \mathrm{MeV}<E<30 \mathrm{MeV}$. The compensated information has the same time resolution as the lower threshold, which was used to generate it. Only at high energies a slight degradation is visible, which results from the clocked operation of the compensation electronics.

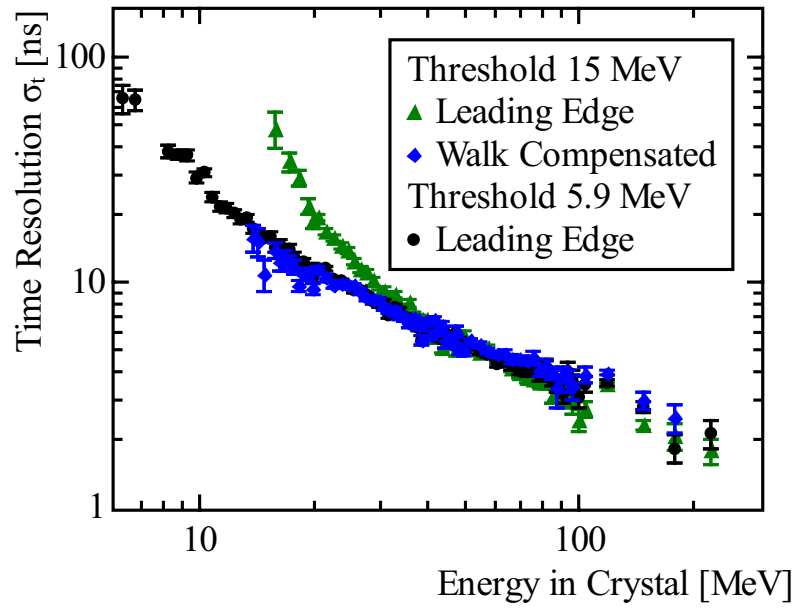

Figure 12. Time resolution of two thresholds and the resolution of the compensated signal [20].

The upcoming SADCs will allow to extract the time information also from the energy channel. A measurement has been performed using a digital oscilloscope to estimate the achievable resolution and threshold. For the measurement, a light pulser was used to generate signals in the front-end. The signal of the pulser was chosen such that the leading edge of the pulse in the timing channel resembles that from scintillation events. Fig. 13 shows

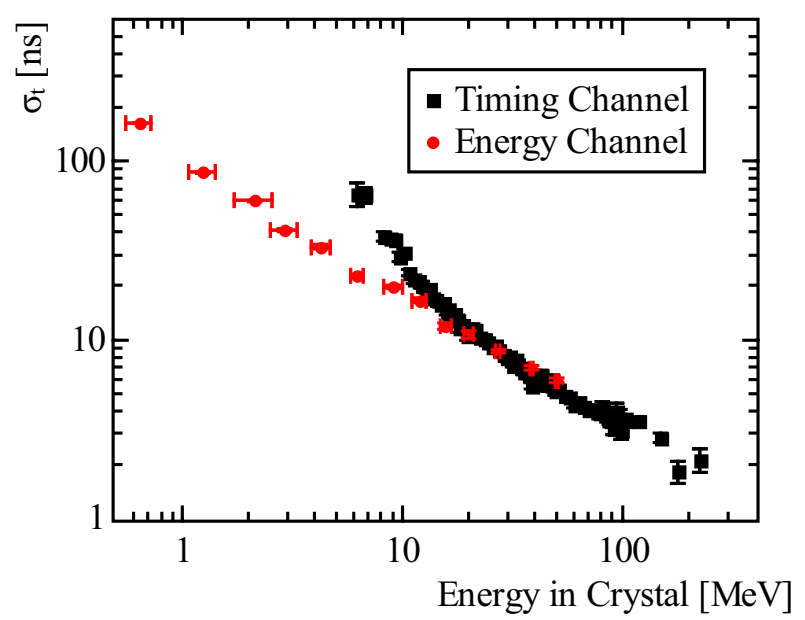

Figure 13. Time resolution of two thresholds and the resolution of the compensated signal [20].

the so found time resolution, compared to the resolution found in the timing channel. The most distinct difference is the much lower detection threshold in the energy channel. The paid price for this improvement is an increased latency of the information. The time was extracted at $50 \%$ of the full amplitude which is $1.5 \mu$ s after the energy deposit. Therefore, the SADCs would not be able to replace the trigger upgrade or the timing channel, still they might provide valuable information for the data analysis.

\subsubsection{Online Gain Monitoring}

The latest prototypes of the sub-detector unit included a light pulser system, which was suited to measure the gain of the APDs during a measurement at a tagged photon beam (see s Sec. 2.4.2). The gain measured during a warm up phase is shown in Fig. 14. Investigations about the

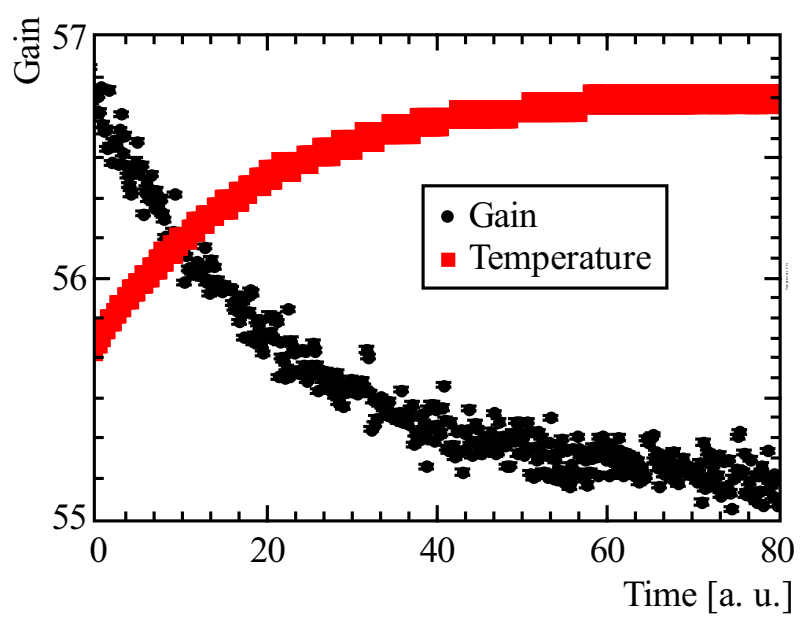

Figure 14. Gain variation during a warm up phase [14].

optimum number of flashes to calculate the gain and the required frequency are ongoing [14].

\subsubsection{Online Gain adjustment}

Although the calorimeter will be operated at a stabilized temperature, a way to improve the gain stabilization to work in a wider temperature range was investigated [19]. Fig. 15 shows the APD gain in dependence of the temperature. The compensation circuit, which is part of the bias supply, stabilizes the gain around the desired operating temperature of $27^{\circ} \mathrm{C}$ (shown as black circles). Adjusting the high voltage setting in the frontend allows to extend the flat region over a much wider temperature range. The method was tested with a single crystal in a temperature stabilized environment. The relative gain was determined using ${ }^{22} \mathrm{Na}$ as reference.

\subsubsection{Status at the end of the SFB}

The calorimeter has been fully upgraded with the new readout. The new backend is being produced. First measurements on a photon beam with a polarized target are planned for 2017. 


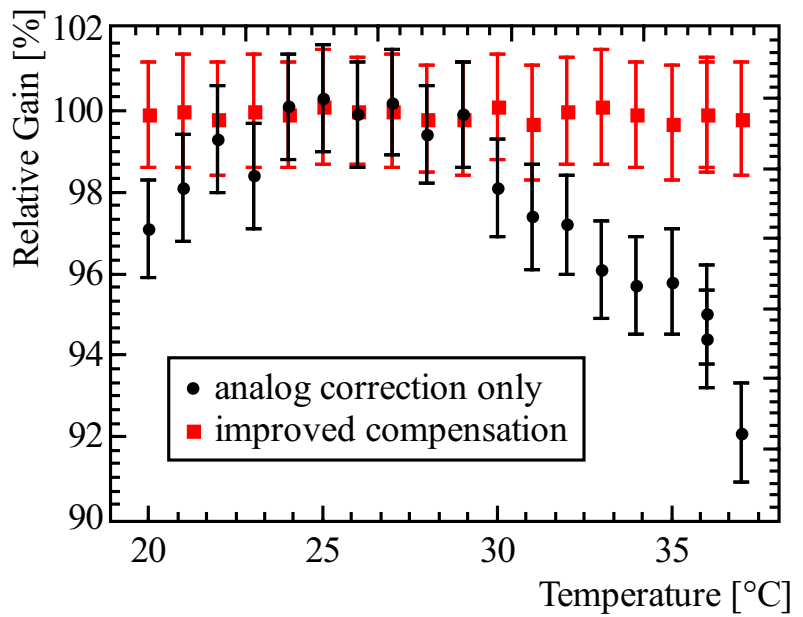

Figure 15. Temperature dependence of the gain. The analog correction shows a flat region at the desired operating temperature (black circles). The improved compensation extends the flat region (red squares) [19].

\section{FPGA-based Sampling-ADC for the Crystal Barrel Calorimeter}

The event-rate of the data acquisition system is currently limited by the digitization stage of the energy branch (LeCroy QDC1885F) to $\approx 2 \mathrm{kHz}$. To overcome this limitation, the implementation of a new Sampling-ADC (SADC) readout is the second important step in the upgrade of the detector system [21].

The prototype design of a 64-channel SADC for the PANDA electromagnetic calorimeter ([23]) was modified, adapting it to the needs of the Crystal Barrel Experiment. The new SADC offers 64 channels in one NIM module with up to 14 bit@80 MHz, accompanied by a custom modular low-noise power supply. Two prototypes are currently under investigation (see Fig 16).

A modular analog input stage allows signal shaping with up to $(\mathrm{CR})^{5}-\mathrm{RC}$ in combination with a digitally adjustable baseline and variable pole-zero compensation [22].

The on-board data processing and reduction is realized with two KINTEX7 FPGAs. Using an FPGA provides a multitude of possibilities for online feature extraction, such as the determination of the energy deposited in the crystal, determination of the timing, and detection and correction of pile-up events.

The readout system will be realized with gigabit ethernet links and COTS switches for the backend, allowing a sufficiently fast readout with feature-extracted data, or alternatively a slower mode in which full samples of a variable length can be transmitted (oscilloscope-mode).

Preliminary measurements have been conducted with a simulated pulse to compare the energy resolution of the SADC to the current LeCroy digitizer ([24]). Different approaches have been compared (see Fig. 17), showing that the SADC performs well. Seemingly, a dual range setup will not be necessary. Two methods are exploited in [24] in which the digitizer is deliberately overdriven,

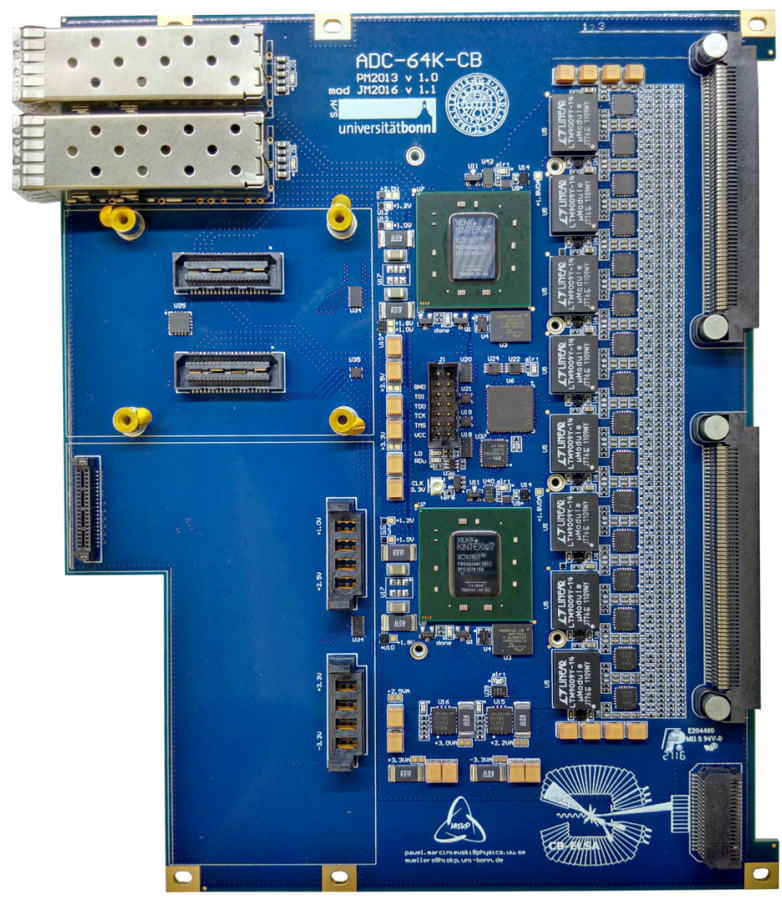

Figure 16. Second Prototype of the CB-SADC board.

allowing a tradeoff between better overall resolution and worse resolution starting with the clipping of the pulse.

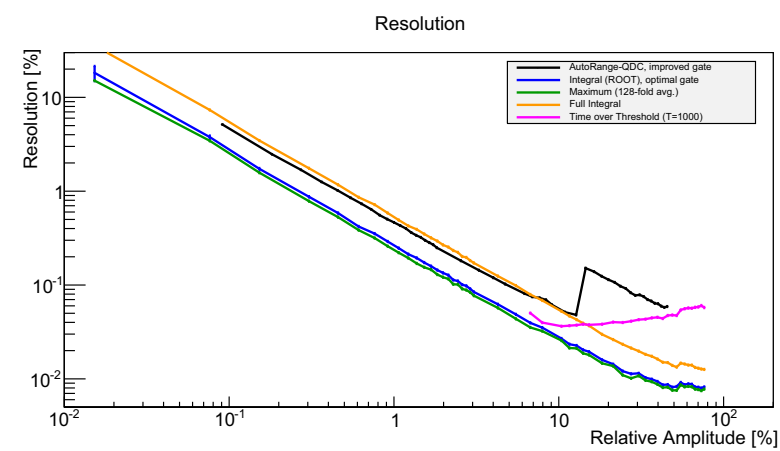

Figure 17. Resolution $(\sigma E / E)$ of different approaches to determine the energy information for the SADC, compared to the resolution of the current dual-range LeCroy digitizer. Also shown is the time-over-threshold method, allowing energy determination for clipped pulses. The $\mathrm{x}$-axis is scaled to the full range of the digitizers.[24]

The prototype setup has been tested under beamconditions in 2016.

\section{MiniTAPS intensity upgrade}

The forward opening of the Crystal Barrel Calorimeter is covered by the TAPS detector system (MiniTAPS), consisting of 216 hexagonal $\mathrm{BaF}_{2}$-crystals of $25 \mathrm{~cm}$ length, individually read out via photomultiplier tubes with bialkali photocathodes and fused silica windows to be sensitive to the $\mathrm{UV}$-components of the $\mathrm{BaF}_{2}$ luminescence. The 
scintillation light consists of two major components based on different mechanisms. The fast component with a decay time of $\tau \sim 0.8 \mathrm{~ns}$ is based on a core-valence transition with emission wavelength at $195 \mathrm{~nm}$ and $210 \mathrm{~nm}$, respectively. Due to this mechanism, the luminescence yield is independent of temperature. The slow component, based on self-trapped excitons, has a decay time of $\tau \sim 620 \mathrm{~ns}$, peaking at an emission wavelength of $320 \mathrm{~nm}$, representing nearly $90 \%$ of the overall scintillation yield. To collect the total scintillation response, a complete integration time of $\sim 2 \mu \mathrm{s}$ is required due to the slow component and is consequently limiting the rate capability of each individual detector component due to pile-up. Since the MiniTAPS device is covering the most forward direction, it has to withstand highest rates. The reduced photon statistics due to the lower light output of the fast component has only a relatively small impact on the achievable resolution as shown in Fig. 18(a), which shows the achieved experimental energy resolution of the reconstructed shower. The concept of the upgrade is based on a hardware suppression of the slow scintillation component. Instead of using a photomultiplier equipped with a solar-blind photocathode, appropriate narrow band UV filters have been placed in between the crystal and photomultiplier. In a detailed study [7], various commercially available filters (see Fig. 18(b)) have been tested with respect to the transmitted absolute light yield and the impact on the resulting low energy threshold and achievable energy resolutions even for low energy $\gamma$-rays. The obtained results are very promising. However, the overall performance can be significantly improved by customized semiconductor-based filters with optimized transmission and dimensions adapted to the detector geometry, which requires development in collaboration with the manufacturer.

Another rate limiting factor is the currently used QDC based TAPS readout electronics which introduces significant deadtime due to its self gating design. To alleviate this situation a new readout based on SADCs is planned.

\section{New Inner Tracker for the Crystal Barrel Detector}

A full scale Time Projection Chamber (TPC) prototype was built for experiments in hadron collisions within the existing FOPI spectrometer at GSI and photoproduction experiments within the Crystal Barrel Calorimeter at ELSA. The prototype is composed of the following parts:

(a) An active gas volume of $48 \mathrm{dm}^{3}$ with a total drift length of $72.78 \mathrm{~cm}$, an inner field cage with a diameter of $10.40 \mathrm{~cm}$ and an outer field cage with a diameter of $30.80 \mathrm{~cm}$. In the cylindrical detection volume, traversing particles ionize the gaseous detection medium producing positive and negative charge carriers in an electrical drift field. This field, which is oriented parallel to the beam axis, is established by a cathode endcap downstream of the target position and the anode layer upstream of it. A field-defining system is required to keep the gradient of the field constant throughout the active volume. All electrode structures, made from metallic material patterned on a carrier material, are optimized in terms of achievable field homogeneity and a low material budget.

(b) An amplification stage that serves to multiply the ionization electrons after they have traversed the drift volume. In case of a GEM-TPC this is achieved by a triple GEM stack of circular shape which is composed of a plane-parallel set of GEM foils oriented perpendicular to the beam axis.

(c) A patterned electrode structure on the anode layer, the pad plane, where the signals corresponding to the collected charges are detected.

(d) The electronic readout based on the AFTER-chip of the $\mathrm{T} 2 \mathrm{~K}$ experiment for the pad plane (10000 channels) and a system of various detector-near sensors monitoring all relevant operation parameters like temperature, pressure and flow of gas and cooling media, high and low voltages.

(e) Gaseous and fluid media lines, low and high voltage supplies and their respective cables, pipes and terminals (panels) needed to operate the TPC, and in addition support, alignment structures and mounting points.

Fig. 19(a) shows a photograph of the full scale prototype that has been built. Furthermore a detailed technical design report (TDR) has been published [2].

\subsection{TPC Beam Telescope and first Test Measurements}

Systematic resolution studies of a GEM-TPC require an excellent external beam reference. For this purpose the TPC tracking beam telescope has been developed [3]. It consists of two planar GEM detectors with a strip readout of $400 \mu \mathrm{m}$ pitch and 2 silicon strip detectors of $50 \mu \mathrm{m}$ pitch which allow for a very precise beam definition of the order of $\sim 25 \mu \mathrm{m}$. Triggering is done with 2 pairs of plastic scintillators of which one pair can be moved with a step motor to perform beam scans to align the whole detector setup. In the middle of the table, a mounting structure is holding a small prototype TPC which was developed to perform reconstruction studies. It has an outer diameter of $20 \mathrm{~cm}$ and a drift length of $7.96 \mathrm{~cm}$. Gas amplification is performed with a triple-GEM-stack and its 1500 pads are read out with 4x6 T2K-AFTER boards. The pad plane consists of two different hexagonal pad types with outer radii of $1.25 \mathrm{~mm}$ and $1.5 \mathrm{~mm}$ which allow resolution studies depending on pad size and pad response. The testbench has been used at the electron beam at ELSA and is currently located at the beam dump of the COMPASS experiment at CERN to optimize the resolution by varying shaping settings and gas parameters in the halo of the high energetic muon beam of $160 \mathrm{GeV}$ with negligible multiple scattering effects. First in-beam tests show a resolution of the track definition of about $25-30 \mu \mathrm{m}$ and a spatial resolution of the TPC of about $200 \mu \mathrm{m}$ with $\mathrm{Ar} / \mathrm{CO}_{2}(70 / 30)$ as a drift gas [3] [5]. To monitor and control all critical TPC parameters like high 


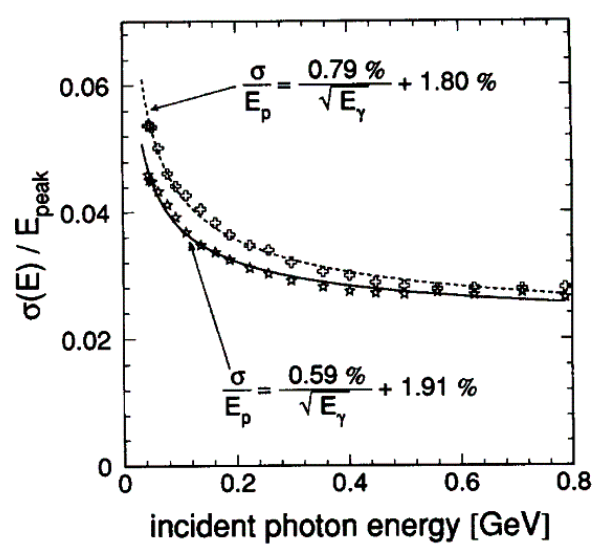

(a)

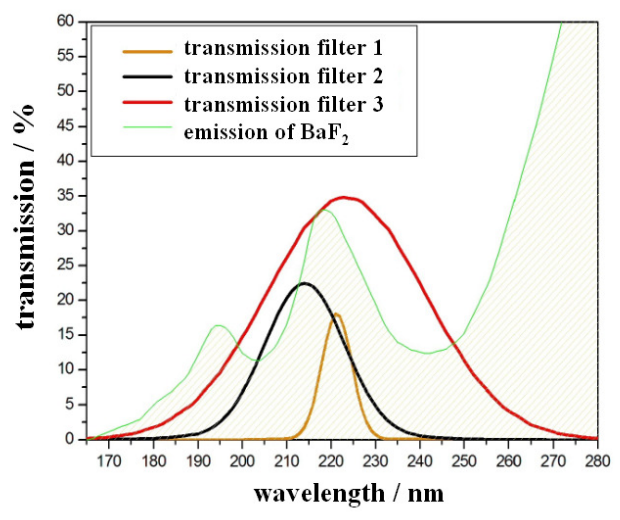

(b)

Figure 18. (a) Energy resolution of a $\mathrm{BaF}_{2}$ sub-matrix of the TAPS detector measured with energy marked photons shown for the total light output (stars) and the fast component (crosses) only (taken from [6]). (b) Optical transmission of three selected optical filters in comparison to the two fast emission components of $\mathrm{BaF}_{2}$. The emission spectrum has been normalized to the transmission of the used filters.

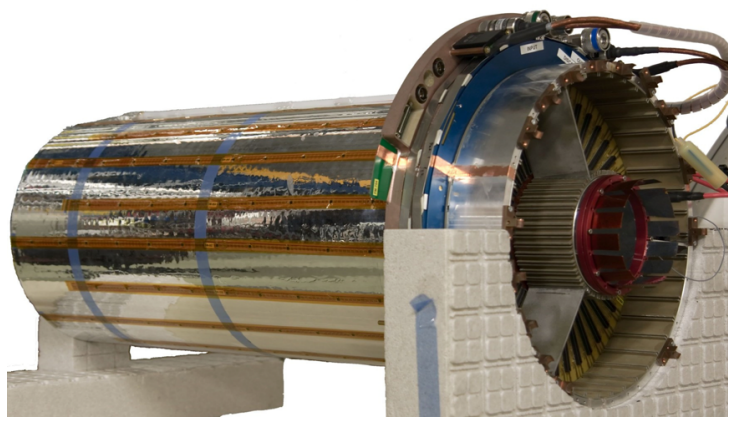

(a)

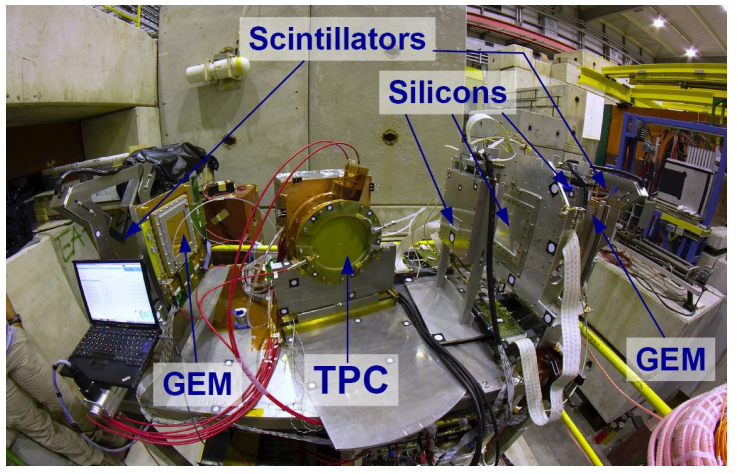

(b)

Figure 19. (a) Photo of the full scale Time Projection Chamber prototype. experiment at CERN. The muon beam is coming from the right side.

(b) Testbench setup on the beam dump platform of the COMPASS

voltages, gas flow and temperatures a fully featured slow control system has been developed. It allows control via a graphical user interface and a web interface, and is designed to easily integrate into other systems, thus allowing for a simple integration of the TPC into existing experiments [13].

\subsection{TPC Energy Calibration}

The preferred method to estimate the energy resolution and perform a relative channel-to-channel calibration for a GEM-TPC in order to improve the overall $\mathrm{dE} / \mathrm{dx}$ resolution is to feed radioactive ${ }^{83 \mathrm{~m}} \mathrm{Kr}$ into the detector. It originates from EC decay of ${ }^{83} \mathrm{Rb}$, mostly populating the isometric state at $41.6 \mathrm{keV}$ which has a half-life of $1.83 \mathrm{~h}$. By this decay different conversion electrons and photons with an energy between $9.4 \mathrm{keV}$ and $41.6 \mathrm{keV}$ are emitted which are detected inside the TPC volume. The calculated energy spectrum from ${ }^{83 \mathrm{~m}} \mathrm{Kr}$ decays taking into account photon conversion factors (for $\mathrm{Ar} / \mathrm{CO}_{2}$ 90/10) inside a TPC is shown in Fig 20(a). A rubidium source with an ac- tivity of $5 \mathrm{MBq}$ has been produced at the HISKP cyclotron in Bonn with a ${ }^{81} \operatorname{Br}(\alpha, 2 \mathrm{n}){ }^{83} \mathrm{Rb}$ reaction (at $54 \mathrm{MeV}$ with $\sigma_{\max }=1200 \mathrm{mb}$ ) and has been integrated into the existing TPC setup with a bypass valve to switch between normal operation and calibration mode. Decay rates of the order of $10^{6}$ per second have been observed inside the GEMTPC, also showing a very stable operation in a high rate environment.

Charge clusters inside the TPC from krypton decays differ significantly from clusters originating from particle tracks in size and overall charge. Nevertheless, inside a magnetic field of $600 \mathrm{mT}$, the clusters in the $\mathrm{x}-\mathrm{y}$ plane are almost point-like or have a circular structure. Therefore the standard peak-finding clustering can be used. A larger cut on cluster length was used, as depending on the effective range inside the drift gas, higher energetic clusters might have a range corresponding to $10-15$ samples for standard settings.

To perform a relative channel-wise calibration, a different method was also tested and compared to the leaderpad method that has been used before. Channel-wise spectra containing the cluster-charge are generated and rela- 


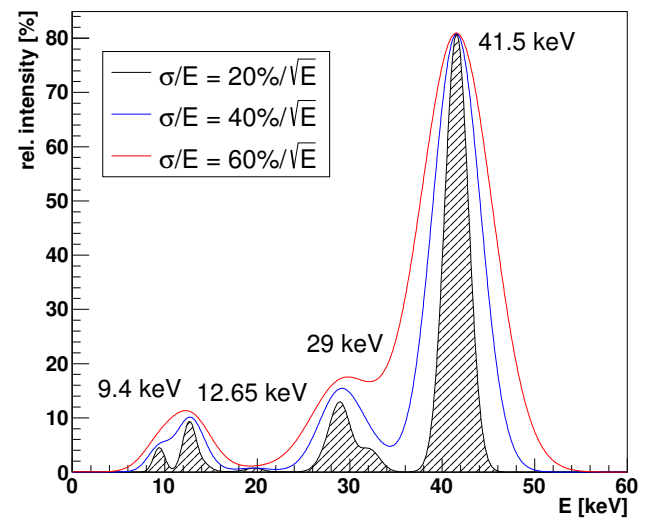

(a)

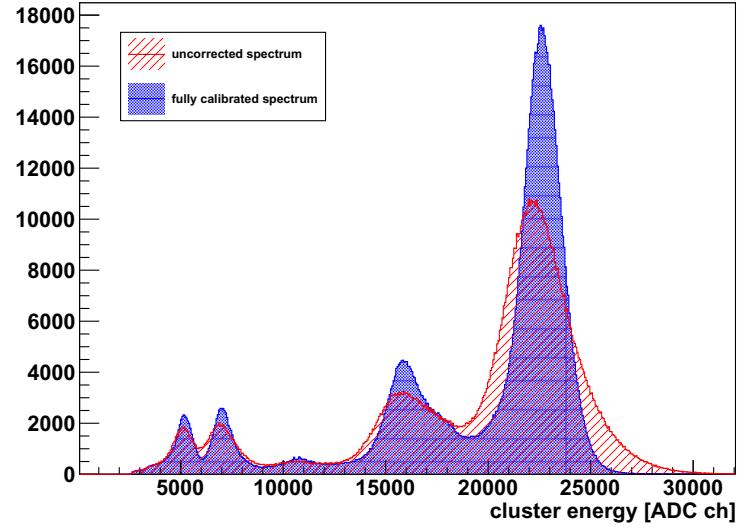

(b)

Figure 20. (a) Theoretical ${ }^{83 \mathrm{~m}} \mathrm{Kr}$ spectrum for different energy resolutions (b) Measured energy distribution before and after calibration.

Table 1. The relative resolutions of the peaks in the krypton spectrum, with and without relative gain calibration.

\begin{tabular}{|c|c|c|}
\hline peak $[\mathrm{keV}]$ & uncorrected & corrected \\
\hline 9.4 & $11.0 \%$ & $6.6 \%$ \\
12.65 & $8.5 \%$ & $6.0 \%$ \\
28.95 & $6.6 \%$ & $4.0 \%$ \\
41.6 & $6.4 \%$ & $3.6 \%$ \\
\hline
\end{tabular}

tive gain factors normalized to the median value of all 10254 channels are calculated by comparing the peak positions for each channel. Using this method, a much faster and more stable convergence was achieved than with the leader-pad method while delivering comparable results. It also simplifies identification of the main peaks as no hard cuts to preselect clusters have to be applied. Relative calibration is done iteratively rerunning the whole reconstruction and recursively correcting the gain factors. In that way a gain uniformity of less than $1 \%$ after 3 iterations can be achieved. [4]

For a first calculation of the energy resolution using the krypton spectrum, the relative width of the peaks was estimated from a fit to the clearly separated peaks, omitting the double-peak structure at $29.0 / 32.3 \mathrm{keV}$, with and without applying relative gain calibration. The spectra after correction show a significant improvement already in the main peak from $6.4 \%$ to $3.6 \%$. The relative resolutions for the single peaks are given in Tab. 1. Taking into account noise and non-uniformities the energy resolution curve follows a $\sigma_{E} / E \sim 0.25 / \sqrt{E}$ function, while after correction $\sigma_{E} / E \sim 0.18 / \sqrt{E}$ was achieved. [5]

The work reported here would not have been possible without the enduring enthusiasm of many bachelor, master, and $\mathrm{PhD}$ students and the continuous technical support from staff members of the participating Universities. The support of the ELSA staff members and the polarized target group is gratefully recognized. We would like to thank C. Honisch, J. Müllers and M. Urban for contributions to this report. We appreciate the support of the
Schweizerischer Nationalfonds, the US National Science Foundation, and the Russian Foundation for Basic Research, in particular the generous support from the Deutsche Forschungsgemeinschaft (SFB/TR16).

\section{References}

[1] I. Jaegle et al. [CBELSA/TAPS Collaboration], "Quasi-free photoproduction of eta-mesons off the deuteron “, Eur. Phys. J. A 47 (2011) 89

[2] M. Ball et al. [GEM-TPC collaboration], "GEMTPC Technical design Report”, arXiv:1207.0013v1 [physics.ins-det] (2012).

[3] A. Winnebeck, "Design studies for a tracking upgrade of the Crystal Barrel experiment and installation of a tracking testbench",

PhD. Thesis HISKP University Bonn (2009).

[4] R. Schmitz [GEM-TPC Collaboration], "Energy calibration of a GEM-TPC Prototype with $\mathrm{Kr}$-83m”, EXA 2011 Conference Record, DOI: 10.1007/s10751-0110555-6 (2012).

[5] R. Schmitz, "Auflösungsstudien und Energiekalibrierung für eine GEM-TPC im Crystal-BarrelExperiment",

PhD. Thesis HISKP University Bonn (2017).

[6] A. R. Gabler et al., "Response of TAPS to monochromatic photons with energies between 45 and $790 \mathrm{MeV}$ ", Nucl. Instr. and Meth. in Phys. Res. A 346 (1994) 168.

[7] S. Diehl, "New Readout Concepts for Electromagnetic Calorimeters", Bachelor Thesis University Gießen (2010).

[8] F.N. Afzal, "Measurement of the double polarization observable E in $\pi^{0}$ - , $\eta$ - and $\eta^{\prime}$ - photoproduction",

PhD. Thesis HISKP University Bonn (2017).

[9] H.v. Pee, private communication (2009).

[10] S. Undorf, "Untersuchungen zur Lichtsammlung von Caesiumiodid-Kristallen",

Bachelor Thesis HISKP University Bonn (2010). 
[11] E. Aker et al., Nucl. Instr. and Meth. in Phys. Res., A 321, 69-109 (1992).

[12] Saint-Gobain Crystals, CsI(Tl), CsI(Na) Cesium Iodide Scintillation Material, Datenblatt, 2007.

[13] D. Kaiser, "Steuerung und Überwachung einer Zeitprojektionskammer mit GEM-Auslese", PhD. Thesis HISKP University Bonn (2014).

[14] M. Urban, "Aufbau eines Lichtpulsersystems zur Messung des Verstärkungsfaktors der APDs in der neuen CB-Ausleseelektronik",

PhD. Thesis HISKP University Bonn (2017).

[15] J. Müllers, "Design and Test of a Slowcontrol for the APD Upgrade of the Crystal Barrel Calorimeter", Master Thesis HISKP University Bonn (2013).

[16] P. Klassen, "Design and Test of a new Cluster Finder for the Crystal Barrel Calorimeter", PhD. Thesis HISKP University Bonn (2017).

[17] J. Bieling, github.com/jobisoft/jTDC.

[18] G. Grutzeck, "Implementierung eines Carry-Chain TDCs auf dem neuen CBDiskriminator Modul", Bachelor Thesis HISKP University Bonn (2016).
[19] P. Pauli, "Studies on an Automatic Gain Stabilisation for the new APD Readout of the Crystal Barrel Calorimeter",

Bachelor Thesis HISKP University Bonn (2015).

[20] C. Honisch "Design, Aufbau und Test einer neuen Ausleseelektronik für das Crystal-Barrel-Kalorimeter", PhD Thesis, HISKP University Bonn (2014).

[21] J. Müllers "An FPGA-based Sampling-ADC Readout for the Crystal Barrel Calorimeter", PhD. Thesis HISKP University Bonn (2017).

[22] T. Poller, "Simulation, Aufbau und Test einer Schaltung zur Baseline-Verschiebung der Crystal Barrel Kalorimetersignale für den Sampling-ADC", Bachelor Thesis HISKP University Bonn (2016).

[23] P. Marciniewski, Angströmlaboratoriet, Uppsala, private communication (2014).

[24] J. Schultes, "Test and Improvement of FeatureExtraction Methods for the new SADC-Readout of the Crystal Barrel Calorimeter", Master Thesis HISKP University Bonn (2016). 